\title{
Actual purchase behavior of edible bird's nest products in Malaysia using cluster analysis
}

\begin{abstract}
The focus of this study was to classify actual buying behavior of edible bird's nest (EBN) products into relatively homogenous groups for the purpose of market segmentation. Determining the profile of actual customers could provide direct characterization of products that should be displayed on the shelf. The data were collected by quota sampling with 312 buyers participated in the survey. Three types of cluster models (K-means, Kohonen, and twostep cluster) were estimated and compared with in order to identify an optimum number of clusters and capture group with a similar pattern. Foremost, a two-step method was chosen for analysis as it provides better average silhouette and well-defined of group segments than the other two cluster methods. The results provide specific characterization of buyers that will be useful to build a target list of potential customers and develop new offerings to meet the new needs of customers for EBN products in Malaysia.
\end{abstract}

Keyword: Consumers' profile; Food safety; Health conscious; Market segmentation; Spending behavior 